Pacific Journal of Mathematics

CHARACTERIZATION OF A CLASS OF TORSION FREE 


\title{
CHARACTERIZATION OF A CLASS OF TORSION FREE GROUPS IN TERMS OF ENDOMORPHISMS
}

\author{
E. F. Cornelius, JR.
}

\begin{abstract}
Characterizations in terms of endomorphisms and quasiendomorphisms are obtained for torsion free abelian groups with the property that each pure subgroup of finite rank is a quasi-summand. A group has this property if and only if its ring of endomorphisms with finite rank is 2-fold ct-transitive, and hence $k$-fold $c t$-transitive for every $k$. This property is equivalent to complete decomposability for countable groups the type set of which satisfies the maximum condition. A stronger version of transitivity is required to describe separable groups the type set of which satisfies the maximum condition; to insure generality, it is shown that the maximum condition does not imply countability of the type set, a result of independent interest.
\end{abstract}

1. Introduction and preliminaries. All groups considered here are subgroups of a fixed vector space $V$ over the rational number field $Q$; we shall refer to these torsion free abelian groups simply as "groups". $G$ will always denote a full subgroup of $V$, i.e., one with torsion quotient $V / G . \quad V$ is thus the divisible hull of $G$ and $r(G)=r(V)$, where $r$ denotes rank. $L(V)$ denotes the algebra of linear transformations of $V . E(G)$ is the endomorphism ring of $G$ and $F(G)$ is the pure ideal of $E(G)$ consisting of all endomorphisms with finite rank. Similarly, $Q E(G)$ is the quasi-endomorphism algebra of $G$ and $Q F(G)$ is the ideal of elements having finite rank. Familiarity with the concept of quasi-isomorphism is assumed; a complete background may be obtained from $[2,3,9,10] . \doteq, \doteq$, $\doteq$ denote quasicontained, quasi-equal, and quasi-isomorphic, respectively. We consider $Q E(G)=\{f \in L(V): f G \leqq G\}$. Since each element of $E(G)$ induces a unique linear transformation on $V$, we regard $E(G) \subseteq Q E(G)$ and use the same symbol to denote an endomorphism of $G$ and also its induced linear transformation. All sums of groups are direct; e.g., notation such as $G \doteq A+B$ implies that $A$ and $B$ are disjoint groups.

We take the following perspective. For $f \in Q E(G)$, define the final rank of $f$ to be the minimum among the cardinal numbers $r\left(f^{n} G\right), n=1,2, \cdots$. We assert the

Proposition 1.1. Each quasi-endomorphism of $G$ with finite positive final rank (especially any such endomorphism of $G$ ) induces a quasi-decomposition of $G$. 
Proof. It suffices to consider $f \in E(G)$ with $0<r\left(f^{n} G\right)=r\left(f^{n+1} G\right)=$ $\cdots<\infty$ for some positive integer n. $f^{n}: f^{n} G \rightarrow f^{n} G$ is thus a quasi-automorphism of $f^{n} G\left[9\right.$, p. 553, Th. 4.2]; suppose $m f^{n} G \cong$ $f^{2 n} G$ for the positive integer $m$. Let $b \in G ; m f^{n} b=f^{2 n} a$ for some $a \in G$. Write $m b=\left(m b-f^{n} a\right)+f^{n} a ;\left(m b-f^{n} a\right) \in \operatorname{ker} f^{n}$ and $f^{n} a \in$ $f^{n} G$; note $\operatorname{ker} f^{n} \cap f^{n} G=0$. Thus $G \doteq \operatorname{ker} f^{n}+f^{n} G$. (We do not exclude the possibility $r(G)=r(f G)<\infty$, i.e., the case when $f$ is a quasi-automorphism of a group with finite rank.)

Thus a group with "many" quasi-endomorphisms of finite final rank has "many" quasi-decompositions. Conversely, we exploit the correspondence between quasi-decompositions of $G$ and idempotents in $Q E(G)[9$, p. 551, Lemma 2.5]. Most proofs here involve the construction of a function which induces some decomposition. We seek to characterize summand properties of groups in terms of a "sufficiency" of mappings. This paper is a continuation of the work begun in [4], where groups in which each pure subgroup of finite rank is a quasi-summand were first studied. There they were described as quasi-separable groups with linearly ordered type sets.

REMARK 1.2. The following conditions on the group $G$ are equivalent:

(1) Each pure subgroup of finite rank in $G$ is a quasi-summand.

(2) Each pure subgroup of rank one in $G$ is a quasi-summand.

(3) $Q E(G)$ contains a projection onto each one-dimensional subspace of $V$.

(4) $Q E(G)$ contains a projection onto each finite-dimensional subspace of $V$.

We frequently employ this equivalence, especially (3); cf. [5]. Each of these properties is inherited by quasi-summands and pure subgroups.

Before pursuing the main results, we prove a lemma of general utility. In order to accomplish this, additional notation is introduced. For $a \in G$, let $t(a)$ or $t_{G}(a)$ denote the type of the element $a$; if $G$ is homogeneous, $t(G)$ also may denote the type of the (nonzero) elements of $G$. Recall that type is invariant under quasi-isomorphism [3, p. 26, Lemma 7.1]. Let $T(G)$ denote the type set of $G$. For the type $\tau$, set $G(\tau)=\{a \in G: t(a) \geqq \tau\}$ and let $G^{*}(\tau)$ be the subgroup of $G$ generated by $\{a \in G: t(a)>\tau\} . G(\tau)$ and $G^{*}(\tau)$ are thus fully invariant subgroups of $G$. If $H$ is a pure subgroup of $G$, then $H(\tau)=H \cap G(\tau)$. Finally, if $G^{*}(\tau)$ is pure in $G$, let $\boldsymbol{G}_{\tau}=G(\tau) / G^{*}(\tau)$.

Lemma 1.3. (1) If $G \doteq A+B$, then $G(\tau) \doteq A(\tau)+B(\tau)$ and 
$G^{*}(\tau) \doteq A^{*}(\tau)+B^{*}(\tau)$, for every type $\tau$.

(2) Suppose $T(G)$ is linearly ordered. If $a$ and $b$ are elements of $G$ such that $t(a)<t(b)$, then $t(a+b)=t(a)$.

(3) If $T(G)$ is linearly ordered, then for every type $\tau, G^{*}(\tau)$ is a pure subgroup of $G$ and $H^{*}(\tau)=H \cap G^{*}(\tau)$ for $H$ pure in $G$.

Proof. (1) holds because type is a quasi-isomorphism invariant and $(A+B)(\tau)=A(\tau)+B(\tau),(A+B)^{*}(\tau)=A^{*}(\tau)+B^{*}(\tau)[1$, p. 75, 3].

In $(2), t(a+b) \geqq t(a)$ and certainly $t(b)>t(a+b)$. Hence

$$
t(a)=t((a+b)-b) \geqq t(a+b) \wedge t(b)=t(a+b) .
$$

(3) now follows from (2).

2. ct-Transitivity. Our immediate goal is to characterize groups with the summand property in terms of endomorphisms. Toward this end, we introduce the concept of $c t$-transitivity.

Definition 2.1. Let $S$ be an independent set of elements in $G$ and suppose that for each type $\tau$, the pure subgroup generated by those elements in $S$ of type $\tau$ is homogeneous. We shall then call $S$ ct-independent.

Ppoposition 2.2. Let $G$ have finite rank $n$. Then $G$ contains a ct-independent set of $n$ elements.

Proof. Set $G_{1}=G$, let $\tau_{1}$ be a maximal type of $G_{1}[6$, p. 148 , Lemma 42.1], and let $\left\{a_{1 j}: j=1, \cdots, k_{1}\right\}$ be a maximal independent subset of $G_{1}\left(\tau_{1}\right)$. Let $G_{2}$ be a subgroup of $G_{1}$ maximal with respect to disjointness from $G_{1}\left(\tau_{1}\right)$, take $\tau_{2}$ to be a maximal type of $G_{2}$, and let $\left\{a_{2 j}: j=1, \cdots, k_{2}\right\}$ be a maximal independent set in $G_{2}\left(\tau_{2}\right)$. By continuing in this fashion, we obtain a $c t$-independent set which is also a maximal independent subset of $G$.

Definition 2.3. A subring $R$ of $E(G)$ is $k$-fold $c t$-transitive if for every $j \leqq k$ the following condition is satisfied: for each $c t$ independent set of elements $a_{1}, \cdots, a_{j}$ in $G$ and each set of elements $b_{1}, \cdots, b_{j}$ in $G$ which satisfy $t\left(a_{i}\right) \leqq t\left(b_{2}\right), i=1, \cdots, j$, some $f \in R$ maps $a_{i}$ to $n_{i} b_{i}, n_{i}$ a positive integer, $i=1, \cdots, j$.

Observe that if $E(G)$ is $k$-fold $c t$-transitive, then so is $E(H)$ for $H$ a quasi-summand or pure, fully invariant subgroup of $G$.

Proposition 2.4. If $E(G)$ is 2-fold ct-transitive, then any two elements of $G$ have comparable types. 
Proof. Suppose the nonzero elements $a$ and $b$ of $G$ have distinct types. If $t(a-b)=t(b)$, then

$$
t(a)=t((a-b)+b) \geqq t(a-b) \wedge t(b)=t(b) .
$$

If $t(a-b)$ and $t(b)$ are distinct, some $f \in E(G)$ maps $a-b$ to 0 and $b$ to $n b, n>0$. Then

$$
t(a) \leqq t(f a)=t(f b)=t(n b)=t(b) .
$$

For $a \in G, a$ denotes the coset of $a$ in some specified quotient group.

LEMMA 2.5. Suppose $T(G)$ is linearly ordered.

(1) A subset $S$ of $G$ is ct-independent if and only if for each type $\tau$, those elements in $S$ of type $\tau$ have cosets independent in $\boldsymbol{G}_{\tau}$.

(2) If $G$ has finite rank $n$, then every ct-independent set can be extended to one containing $n$ elements.

(3) Let $G$ be completely decomposable of finite rank $n$ and let $a_{1}, \cdots, a_{n}$ be ct-independent in $G$. If $A_{i}$ denotes the pure subgroup generated by $a_{i}$, then $G \doteq A_{1}+\cdots+A_{n}$.

Proof. By Lemma 1.3 (3), $G^{*}(\tau)$ is a pure subgroup of $G$, so (1) holds.

For (2), let $S$ be a $c t$-independent subset of $G$ and suppose $T(G)=$ $\left\{\tau_{1}, \cdots, \tau_{l}\right\}\left[6\right.$, p. 148, Lemma 42.1]. Let $S_{i}=\left\{a_{i j}: j=1, \cdots, l_{i}\right\}$ be those elements in $S$ of type $\tau_{i}, i=1, \cdots, l$. By (1) above, $\left\{\boldsymbol{a}_{i j}: j=\right.$ $\left.1, \cdots, l_{i}\right\}$ is an independent set in $\boldsymbol{G}_{\tau_{i}}$, which can be extended to a maximal independent subset of $\boldsymbol{G}_{\tau_{i}},\left\{\boldsymbol{a}_{i j}: a_{i j} \in G\left(\tau_{i}\right), j=1, \cdots, m_{i}\right\}, i=$ $1, \cdots, l$. Now $\left\{a_{i j}: j=1, \cdots, m_{i} ; i=1, \cdots, l\right\}$ is a $c t$-independent set containing $S$, and it is also a maximal independent subset of $G$.

In (3), we may assume $t\left(A_{1}\right) \leqq \cdots \leqq t\left(A_{n}\right)$. For nonzero $b \in A=$ $A_{1}+\cdots+A_{n}$, write $b=b_{j}+\cdots+b_{n}, b_{i} \in A_{i}, i=j, \cdots, n$, with $b_{j} \neq 0$. Then $t_{A}(b)=t_{A}\left(b_{j}\right)=t_{G}\left(b_{j}\right)=t_{G}(b)$; the last equality holds by Lemma $1.3(2)$. The conclusion now follows from [1, p. 97, Lemma 7.1(a)].

DEFINITION 2.6. A subring $R$ of $E(G)$ is called $k$-fold transitive if for every $j \leqq k$ independent elements $a_{1}, \cdots, a_{j}$ of $G$ and $j$ arbitrary elements $b_{1}, \cdots, b_{j}$ in $G$, there exists $f \in R$ such that $f a_{i}=$ $n_{i} b_{i}, n_{i}$ a positive integer, $i=1, \cdots, j$.

REMARK 2.7. (1) Let $R, a_{1}, \cdots, a_{j}$, and $b_{1}, \cdots, b_{j}$ be as in Definition 2.3 or 2.6. Then there exists $f \in R$ such that $f a_{i}=n b_{i}$, 
$i=1, \cdots, j$, for a single positive integer $n$. For by definition, there exists $f_{i} \in R$ such that $f_{i} a_{i}=m_{i} b_{i}, m_{i}>0$, and $f_{i} a_{l}=0$ for $l \neq i$, $i=1, \cdots, j$. Now $f=\sum_{i=1}^{j} \prod_{l \neq i} m_{l} f_{i} \in R$ satisfies $f a_{i}=\left(m_{1} \cdots m_{j}\right) b_{i}$, $i=1, \cdots, j$.

(2) Definition 2.6 is thus consistent with [4, p. 610, Def. 3.5].

(3) We retain the terminology in Definition 2.6 for two reasons. First, it is the correct interpretation in terms of group endomorphisms of $k$-fold transitivity in $L(V)$ [4, p. 610, Prop. 3.6]; our definition of transitivity in $L(V)$ is standard [8, p. 32]. Secondly, we want all properties of $G$, such as homogeneity, to be consequences of properties of $E(G)$. This latter desire motivates our definition of $c t$-transitivity in terms of $c t$-independent sets.

(4) It might seem more natural to define $c t$-transitivity in terms of "quasi-pure independent" sets, i.e., independent sets $a_{1}, \cdots, a_{j}$ such that the pure subgroup generated by all the $a_{i}$ 's is quasi-equal to the direct sum of the pure subgroups generated by the individual elements. By Lemma $2.5(3)$, if $T(G)$ is linearly ordered, then a ctindependent set is in fact "quasi-pure independent", and comparability of types is a consequence of 2 -fold ct-transitivity, by Proposition 2.4. However, there exists an abundance of groups in which the only "quasi-pure independent" sets consist of single elements, e.g., pure subgroups of the $p$-adic integers. For pathological examples even among groups of rank two, consult [3, pp. 28-30]. Proposition 2.2 guarantees the existence of nontrivial $c t$-independent sets.

Lemma 2.8. If $R$ is a 2-fold ct-transitive subring of $E(G)$, then $R$ induces a 2-fold transitive subring $\boldsymbol{R}$ in $E\left(\boldsymbol{G}_{\tau}\right)$, for all relevant types $\tau . \quad \boldsymbol{G}_{\tau}$ is thus homogeneous and $\boldsymbol{R}$ is $k$-fold transitive for every $k$.

Proof. That $R$ induces a subring in $E\left(G_{\tau}\right)$ follows from Proposition 2.4, Lemma 1.3(3), and the full invariance of $G(\tau)$ and $G^{*}(\tau)$. To see that $\boldsymbol{R}$ is 2 -fold transitive, let $\boldsymbol{a}_{1}, \boldsymbol{a}_{2}$ be independent in $\boldsymbol{G}_{\tau}$ and let $\boldsymbol{b}_{1}, \boldsymbol{b}_{2}$ be arbitrary, $a_{1}, a_{2}, b_{1}, b_{2}$ all in $G(\tau)$. By Lemma $2.5(1), a_{1}$ and $a_{2}$ are $c t$-independent (of type $\tau$ ) and $t\left(a_{i}\right) \leqq t\left(b_{i}\right), i=1,2$. By hypothesis, some $f \in R$ maps $a_{i}$ to $n_{i} b_{i}, n_{i}>0, i=1$, 2 , so the induced map sends $\boldsymbol{a}_{i}$ to $n_{i} \boldsymbol{b}_{i}, i=1,2 . \quad \boldsymbol{R}$ is thus 2 -fold transitive. By [4, p. 610, Prop. 3.6] and [4, p. 607, Remark 2.1], $R$ is $k$-fold transitive for every $k$.

We proceed to show that 2 -fold $c t$-transitivity implies $k$-fold $c t$ transitivity for every $k$.

Lemma 2.9. Let $R$ be a 2-fold ct-transitive ring of endomor- 
phisms of $G$ and let $a_{1}, \cdots, a_{k}$ be ct-independent elements of $G$ with $t\left(a_{1}\right) \leqq \cdots \leqq t\left(a_{k}\right)$. Then there exists $f \in R$ such that $f a_{1}=n a_{1}, n a$ positive integer, and $f a_{i}=0, i=2, \cdots, k$.

Proof. Suppose we have established this for $k-1$ ct-independent elements, $k-1 \geqq 2$, and let $a_{1}, \cdots, a_{k}$ be $k$ ct-independent elements arranged so that $t\left(a_{1}\right) \leqq \cdots \leqq t\left(a_{k}\right)$. We distinguish two cases; first, $t\left(a_{k}\right)>t\left(a_{1}\right)$. By our induction hypothesis, there exists $f \in R$ sending $a_{1}$ to $n a_{1}$ and $a_{i}$ to $0, i=2, \cdots, k-1$. If $f a_{k}=0, f$ suffices. If $f a_{k} \neq 0$, then $t\left(a_{1}\right)<t\left(a_{k}\right) \leqq t\left(f a_{k}\right)$, so by the 2-fold ct-transitivity of $R$, some $g \in R$ maps $a_{1}$ to $m a_{1}, m>0$, and $f a_{k}$ to 0 . Now $g f$ has the desired property. Secondly, if $t\left(a_{k}\right)=t\left(a_{1}\right)=\tau$, we consider the ring $\boldsymbol{R}$ induced by $R$ in $E\left(\boldsymbol{G}_{\tau}\right)$. By Lemma 2.5 (1), the cosets $\boldsymbol{a}_{1}$, $\cdots, \boldsymbol{a}_{k}$ are independent in $\boldsymbol{G}_{\tau}$. By Lemma 2.8, some $\boldsymbol{f} \in \boldsymbol{R}$ maps $\boldsymbol{a}_{1}$ to $n a_{1}$ and $a_{i}$ to $0, i=2, \cdots, k$. If $f \in R$ induces $f$, we have $f a_{1}=$ $n a_{1}+b_{1}, b_{1} \in G^{*}(\tau)$, and $f a_{i}=b_{i}, b_{i} \in G^{*}(\tau), i=2, \cdots, k$. Let $B$ be the pure subgroup of $G$ generated by $b_{1}, \cdots, b_{k}$; if $B=\{0\}, f$ suffices. Otherwise, by Proposition 2.2, there exist $c_{1}, \cdots, c_{m}$ ct-independent elements of $B$ with $m=r(B)$. We now apply the previous case to $a_{1}, c_{1}, \cdots, c_{m}$ (even if $m=k$ ). There exists $g \in R$ such that $g a_{1}=l a_{1}$ and $g c_{i}=0, i=1, \cdots, m ; g f$ has the desired property.

THEOREM 2.10. If $R$ is a 2-fold ct-transitive subring of $E(G)$, then $R$ is k-fold ct-transitive for every $k$.

Proof. Assume $R$ is $(k-1)$-fold $c t$-transitive for $k-1 \geqq 2$, let $a_{1}, \cdots, a_{k}$ be $c t$-independent in $G$, and let $b_{1}, \cdots, b_{k}$ be elements of $G$ satisfying $t\left(a_{i}\right) \leqq t\left(b_{i}\right), i=1, \cdots, k$. By Proposition 2.4, we may assume $t\left(a_{1}\right) \leqq \cdots \leqq t\left(a_{k}\right)$. By the induction hypothesis, some $f \in R$ satisfies $f a_{i}=n_{i} b_{i}, n_{i}>0, i=2, \cdots, k$. Lemma 2.9 shows that there exists $g \in R$ such that $g a_{1}=n a_{1}, g a_{i}=0, i=2, \cdots, k$. Finally, if $h \in R$ maps $a_{1}$ to $m\left(b_{1}-f a_{1}\right)$, then $h g+m n f \in R$ sends $a_{1}$ to $(m n) b_{1}$ and $a_{\imath}$ to $\left(m n n_{i}\right) b_{i}, i=2, \cdots, k$.

Proposition 2.11. If $E(G)$ is 2-fold ct-transitive, then every pure subgroup of finite rank in $G$ is completely decomposable.

Proof. Let $A$ be a pure subgroup of finite rank in $G$; we show that each pure subgroup $B$ of rank one in $A$ is a quasi-summand of $A$. Let $b_{1}$ be a nonzero element of $B$. According to Proposition 2.4 and Lemma $2.5(2), T(G)$ is linearly ordered so $b_{1}$ may be extended to a $c t$-independent set $b_{1}, \cdots, b_{n}$ which is a maximal independent set in $A$. By Theorem 2.10, there exists an endomorphism $f$ of $G$ such that $f b_{1}=m b_{1}, m>0$, and $f b_{i}=0, i=2, \cdots, n$. Observe that 
that the restriction of $f$ to $A$ is an endomorphism of $A$ and thus $\mathrm{f} / \mathrm{m}$ is a quasi-endomorphism making $B$ a quasi-summand of $A$ [4, p. 607, Lemma 2.2]. The complete decomposability of $A$ now follows from [4, p. 605, Lemma 1.5] and [4, p. 605, Th. 1.6].

COROLlaRY 2.12. If $E(G)$ is 2-fold ct-transitive, then every countable, homogeneous, pure subgroup $H$ of $G$ is completely decomposable.

Proof. By Proposition 2.11, every pure subgroup of finite rank in $H$ is completely decomposable. The conclusion follows from the homogeneity of $H$ and [4, p. 609, Lemma 3.1].

We now relate $c t$-transitivity in $E(G)$ to density in the finite topology of $Q E(G)$.

REMARK 2.13. Let $R$ be a subring of $L(V)$ and equip $R$ with the finite topology [8, p. $28, \mathrm{Ch}$. II $\S 3]$. Since $G$ is full in $V$, a basis for the neighborhood system of $f \in R$ consists of all sets of the form $\{g \in R: g a=f a$ for all $a \in H\}$, where $H$ is a pure subgroup of finite rank in $G$. Obviously, if $f$ and $g$ agree on a maximal independent set in $H$, then they are equal on all of $H$.

Throughout this paper, topological terms refer to the finite topology of some specified ring of endomorphisms. For a subring $R$ of $E(G), Q R$ denotes the subalgebra of $Q E(G)$ generated by $R$. Recall [4, p. 604, Def. 1.1] that $G$ is quasi-separable if every finite subset of $G$ is contained in a completely decomposable quasi-summand; recently it has been shown sufficient for each single element to be so contained [5].

Lemma 2.14. (1) If $R$ is a dense subring of $E(G)$, then $Q R$ is dense in the finite topology of $Q E(G)$.

(2) Let $R$ be a subring of $E(G)$ and suppose that $E(G)$ is 2-fold ct-transitive. Then $R$ is 2-fold ct-transitive if and only if $Q R$ is dense in $Q E(G)$.

(3) $Q F(G)$ is dense in $Q E(G)$ if and only if each finite subset of $G$ is contained in a quasi-summand of finite rank.

Proof. For (1), let $f \in Q E(G)$ and let $H$ be a pure subgroup of finite $\operatorname{rank}$ in $G$. Suppose $n f \in E(G)$ for $n>0$. By hypothesis, there exists $g \in R$ which agrees with $n f$ on $H$. Then $g / n \in Q R$ agrees with $f$ on $H$, and so $Q R$ is dense in $Q E(G)$.

In (2), the sufficiency is straightforward. For the converse, let 
$f, H$, and $n$ be as in the proof of (1). By Proposition 2.2, $H$ contains a $c t$-independent set $a_{1}, \cdots, a_{m}$ which is also a maximal independent set in $H$. By hypothesis and Remark 2.7 (1), there exists $g \in R$ such that $g a_{i}=l\left((n f) a_{i}\right), i=1, \cdots, m$, for some positive integer $l$. Thus $g /(\ln ) \in Q R$ agrees with $f$ on $H$, and so $Q R$ is dense in $Q E(G)$.

In (3), the sufficiency is likewise clear. To establish the necessity, it suffices to show that each pure subgroup $H$ of finite rank in $G$ is contained in a quasi-summand of finite rank. By density, there exists $f \in Q F(G)$ which agrees on $H$ with the identity mapping of $G$. As in Proposition 1.1, $f$ induces a quasi-decomposition of $G$ with $H$ contained in a quasi-summand of finite rank.

CoROLlary 2.15. If $Q F(G)$ is dense in $Q E(G)$ and every pure subgroup of finite rank in $G$ is completely decomposable, then $G$ is quasi-separable.

Proof. By Lemma 2.14 (3), each finite subset of $G$ is contained in a quasi-summand of finite rank, which may be assumed to be a pure subgroup of $G[2$, p. 95 , Lemma 9.4], and so completely decomposable. $G$ is thus quasi-separable.

REMARK 2.16. (1) The converse of Lemma 2.14 (1) is false. Consider the group $S[4$, p. 607] in the light of [4, p. 611, Th. 3.10 (4)]. Obviously $F(S)$ cannot be dense in $E(S)$.

(2) The converse of Corollary 2.15 is also false, since there exist completely decomposable groups of finite rank which contain indecomposable pure subgroups [6, p. 166].

3. Main theorems. By using the machinery developed in $\S 2$, we now prove the principal results.

THEOREM 3.1. The following conditions on the group $G$ are equivalent:

(1) Each pure subgroup of finite rank in $G$ is a quasi-summand.

(2) $G$ is quasi-separable with linearly ordered type set.

(3) $F(G)$ is 2-fold ct-transitive, and hence k-fold ct-transitive for every $k$.

(4) $T(G)$ forms a chain, every pure subgroup of finite rank in $G$ is completely decomposable, and $Q F(G)$ is dense in the finite topology of $Q E(G)$.

(5) $T(G)$ forms a chain, every pure subgroup of finite rank in $G$ is completely decomposable, and $F(G)$ is one-fold ct-transitive.

(6) For each pure subgroup $H$ of finite rank in $G$, there exists an endomorphism $f$ of $G$ such that $f G=H$ and $f H \neq\{0\}$. 
Proof. As mentioned previously, the equivalence of (1) and (2) is established in [4, p. 606, Cor. 1.7]; it is included here for the sake of completeness.

To see that (1) implies (3), let $a_{1}, a_{2}$ be $c t$-independent elements of $G$ and let $b_{1}, b_{2}$ be elements of $G$ satisfying $t\left(a_{i}\right) \leqq t\left(b_{i}\right), i=1,2$. Let $A_{1}, A_{2}, B_{1}, B_{2}$, and $A$ denote the pure subgroups of $G$ generated by $a_{1}, a_{2}, b_{1}, b_{2}$, and $A_{1}+A_{2}$, respectively. By hypothesis, $A$ is a quasi-summand of $G$ and $A \doteq A_{1}+A_{2}$, by Lemma 2.5 (3); suppose $G \doteq A_{1}+A_{2}+C$ with $n G \cong A_{1}+A_{2}+C$ for $n>0$. Let $e_{1}$ and $e_{2}$ be the idempotents in $E\left(A_{1}+A_{2}+C\right)$ projecting $A_{1}+A_{2}+C$ onto $A_{1}$ and $A_{2}$, respectively. By [4, p. 611, Lemma 3.9], there are maps $f_{i}: A_{i} \rightarrow B_{i}$ sending $a_{i}$ to $n_{i} b_{i}$ for some positive integer $n_{i}, i=1,2$. Now $\left(f_{1} e_{1}+f_{2} e_{2}\right) n \in F(G)$ maps $a_{i}$ to $\left(n n_{i}\right) b_{i}, i=1,2$, so $F(G)$ is 2 -fold $c t$-transitive, and hence $k$-fold $c t$-transitive for every $k$ by Theorem 2.10.

That (3) implies (2) follows from Propositions 2.4, 2.11, Lemma $2.14(2)$ and (3), and Corollary 2.15.

A proof that (3) and (4) are equivalent is implicit in the preceding arguments.

The equivalence of (1)-(4) shows that (1) implies (5).

Conversely, assume (5). Let $U$ be a one-dimensional subspace of $V$ and let $a$ be a nonzero element of $A=U \cap G$. Let $f \in F(G)$ send $a$ to a positive multiple of itself, $n a$, and let $B$ be the pure subgroup of $G$ generated by $f G$. By assumption, $B$ is completely decomposable with linearly ordered type set and so $A$ is a quasi-summand [4, p. 605, Th. 1.6], $B \doteq A+C$. Suppose $m B \subseteq A+C$ for $m>0$ and let $e \in E(A+C)$ project $A+C$ onto $A$. Then $e f / n \in Q E(G)$ projects $V$ onto $U$. According to Remark 1.2 (3), (5) implies (1).

In turn, suppose (1) holds. Let $H$ be a pure subgroup of finite rank in $G$; by hypothesis $H$ is a quasi-summand and in fact a completely decomposable one [4, p. 606, Cor. 1.7], say $G \doteq H+C$. Suppose $n G \leqq H+C$ for $n>0$ and let $e$ denote the projection of $H+C$ onto $H$. Observe that $e(n G) \cong H$ [2, p. 96, Cor. 9.6] via some map $g$; gen is then an epimorphism of $G$ upon $H$ which is nonzero on $H$.

Finally, we show (6) implies (1). Let $U$ be a one-dimensional subspace of $V$. By assumption, there exists an endomorphism $f$ of $G$ which sends $G$ onto $A=G \cap U$ with $f A \neq\{0\}$. Let $a$ be a nonzero element of $A$ and suppose $f a=\lambda a, \lambda$ a nonzero rational number. Now $f / \lambda \in Q E(G)$ is a projection of $V$ onto $U$, so (1) is true by Remark $1.2(3)$.

CoRollary 3.2. Suppose that $G$ satisfies any (and hence all) of the conditions of Theorem 3.1. Then $\boldsymbol{G}(\tau)$ is homogeneous (of type $\tau$ ) and quasi-separable, for each $\tau \in T(G)$. 
Proof. That $G(\tau)$ is homogeneous and quasi-separable follows from Theorem 3.1, Lemma 2.8, and [4, p. 610, Remark 3.7 (4)]. It remains to show that each nonzero element has type $\tau$. Pick $a \epsilon$ $G(\tau), a \notin G^{*}(\tau)$. Let $A$ be the pure subgroup of $G$ generated by $a$ and let $H$ be that pure subgroup generated by $a$ and $G^{*}(\tau)$. Since the summand property is inherited by pure subgroups, $H \doteq A+G^{*}(\tau)$. Thus $H / G^{*}(\tau) \doteq A$, so $\boldsymbol{a}$ has type $\tau$ in $\boldsymbol{G}(\tau)$ because type is invariant under quasi-isomorphism [3, p. 26, Lemma 7.1].

In $[4$, p. 610 , Remark $3.7(5)]$, it is proved that a countable group $G$ is homogeneous and completely decomposable if and only if $E(G)$ is 2 -fold transitive. This result can be extended to a class of countable groups with 2 -fold ct-transitive endomorphism rings.

Lemma 3.3. Let $E(G)$ be 2-fold ct-transitive and suppose that for the type $\tau, G_{\tau}$ is countable and homogeneous of type $\tau$. Then $G^{*}(\tau)$ is a direct summand of $G(\tau)$.

Proof. By Lemma 2.8, $E\left(\boldsymbol{G}_{\tau}\right)$ is 2-fold transitive and so completely decomposable, by the aforementioned result on countable homogeneous groups. The hypothesis that the nonzero elements of $\boldsymbol{G}_{\tau}$ have type $\tau$ and [1, p. 107, Cor. 8.7] yield the conclusion.

LEMmA 3.4. Let $G$ be countable and let $E(G)$ be 2-fold ct-transitive. Suppose further that $T(G)$ satisfies the ascending chain condition and that $\boldsymbol{G}_{\tau}$ is homogeneous of type $\tau$, for each relevant type $\tau$. Then $G$ is completely decomposable.

Proof. By Lemma 3.3, $G(\tau)=G_{\tau}+G^{*}(\tau)$ with $G_{\tau}$ a countable, completely decomposable group, for each relevant type $\tau$. By $[1, p$. 109, Th. 9.3], $G=\sum G_{\tau}(\tau \in T(G))$.

THEOREM 3.5. Let $G$ be a countable group such that $T(G)$ satisfies the ascending chain condition. Then $G$ satisfies any (and hence all) of the conditions of Theorem 3.1 if and only if $G$ is completely decomposable with linearly ordered type set.

Proof. Only the necessity need be proved. By Lemma 3.4, it suffices to demonstrate that $G_{\tau}$ is homogeneous of type $\tau$, for all $\tau \in T(G)$, and this has been done in Corollary 3.2.

COROLLARY 3.6. Let $G$ satisfy any one of the conditions of Theorem 3.1 and let $H$ be a countable pure subgroup or quasisummand of $G$. If $T(H)$ satisfies the ascending chain condition, 
then $H$ is completely decomposable.

4. Applications to separable groups. Theorem 3.1 can be extended along the lines of Theorem 3.5 to describe a class of separable groups in which each pure subgroup of finite rank is a quasi-summand. In order to utilize a theorem of R. Baer [1, p. 117, Th. 11.3], we need a series of preliminary results. We continue to construct functions which induce decompositions.

Recall [1, p. 80, Def. 5.1] that $a \in G$ is primitive of type $\tau$ if $t_{G}(a)=\tau$ and $h_{G}(a)=h_{G(\tau)}\left(a+G^{*}(\tau)\right)$. A finite subset of $G$ is primitive if its elements are primitive with different types.

LEMMA 4.1. Let $F(G)$ be 2-fold ct-transitive and suppose that for each pair $a, b$ of primitive elements having equal height in $G$, there exists an endomorphism of $G$ which maps a to $b$. Then $G(\tau)$ is homogeneous (of type $\tau$ ) and separable, for all $\tau \in T(G)$.

Proof. By [5, Cor.], it suffices to prove that each pure subgroup $\boldsymbol{H}$ of rank one in $\boldsymbol{G}(\tau)$ is a direct summand. Now $\boldsymbol{G}(\tau) \doteq \boldsymbol{H}+\boldsymbol{C}[4$, p. 606, Cor. 1.7] and in fact $\boldsymbol{G}(\tau)=\boldsymbol{H}^{\prime}+\boldsymbol{C}[2$, p. 96, Cor. 9.6] with $\boldsymbol{H} \cong \boldsymbol{H}^{\prime}$ via $\boldsymbol{g}$, provided $\boldsymbol{C}$ is pure in $\boldsymbol{G}(\tau)$ (and we take it to be [2, p. 95, Lemma 9.4]). Suppose $\boldsymbol{H}=H / G^{*}(\tau)$ and $\boldsymbol{H}^{\prime}=H^{\prime} / G^{*}(\tau)$ with $H$ and $H^{\prime}$ pure subgroups of $G$. By [7, p. 114, Prop. 86.5], $H=$ $A+G^{*}(\tau), H^{\prime}=A^{\prime}+G^{*}(\tau)$ for some subgroups $A, A^{\prime}$ of $G$. Let $a$ and $a^{\prime}$ be nonzero elements of $A$ and $A^{\prime}$, respectively, such that $h_{G}(a)=h_{G}\left(a^{\prime}\right)$; note that $a$ and $a^{\prime}$ are primitive. We may thus assume $\boldsymbol{g}(\boldsymbol{a})=\boldsymbol{g}\left(\boldsymbol{a}^{\prime}\right)$ [4, p. 611, Lemma 3.9]. By hypothesis, there exists $f \in E(G)$ such that $f a=a^{\prime}$; denote by $\boldsymbol{f}$ the endomorphism of $G(\tau)$ induced by $f$. Let $\boldsymbol{e} \in E(\boldsymbol{G}(\tau))$ project $\boldsymbol{G}(\tau)$ onto $\boldsymbol{H}^{\prime}$. Now gef projects $\boldsymbol{G}(\tau)$ onto $\boldsymbol{H}$, so $\boldsymbol{H}$ is a direct summand.

LeMma 4.2. Suppose $G$ is quasi-separable with linearly ordered type set and let $H$ denote a subgroup of $G$ such that (1) $H$ is homogeneous of type $\tau$ and (2) $H$ is a direct summand of $G(\tau)$. If each subgroup of rank one in $G$ which satisfies (1) and (2) is a direct summand of $G$, then the same holds for each subgroup of finite rank.

Proof. Suppose we have shown that each subgroup of rank $\leqq n$ which satisfies (1) and (2) is a direct summand of $G, n \geqq 1$, and let $H$ be a subgroup of rank $n+1$ which satisfies (1) and (2). By [4, p. 606, Cor. 1.7], $H$ is completely decomposable so we may write $H=A+B$ with $A$ of rank $n$. By hypothesis, $G=A+C$ and so $G(\tau)=A+C(\tau)$ and $H=A+H \cap C(\tau)$. Again, $H \cap C(\tau)$ is a direct summand of $G, G=H \cap C(\tau)+B$, so $C=H \cap C(\tau)+B \cap C$. Upon 
combining results, we have $G=H+B \cap C$.

The following restrictions on a group and its endomorphisms are sufficient for the group to be separable.

Proposition 4.3. Assume $T(G)$ satisfies the ascending chain conidition. Let $F(G)$ be 2-fold ct-transitive and suppose that for each pair of primitive elements $a, b$ which have equal height in $G$, there exists $f \in F(G)$ such that $f a=b$. Then $G$ is separable.

Proof. By Lemmas 4.1, 4.2, and [1, p. 117, Th. 11.3], it suffices to show that for each $\tau \in T(G)$, every subgroup $A$ of rank one in $G$ which satisfies $t(A)=\tau$ and $G(\tau)=A+C$, is a direct summand of $G$. Let $a$ be any nonzero element of $A$; note that $a$ is primitive. By assumption, some $f \in F(G)$ maps $a$ to $a$. Let $H$ be the pure subgroup of $G$ generated by $f G ; H$ is completely decomposable [4, p. 606, Cor. 1.7]. $a$ is certainly primitive in $H$ and so $H=A+B$ [1, p. 80, Lemma 5.2]. If $e \in E(H)$ projects $H$ onto $A$, then ef $\in E(G)$ projects $G$ onto $A$.

Definition 4.4. Let $R$ be a $k$-fold $c t$-transitive subring of $E(G)$. $R$ is said to be fully $k$-fold $c t$-transitive if for each $j \leqq k$ the following holds: for each primitive set $a_{1}, \cdots, a_{j}$ of elements in $G$ and each set $b_{1}, \cdots, b_{j}$ of elements in $G$ which satisfy $h\left(a_{i}\right) \leqq h\left(b_{i}\right), i=1, \cdots, j$, there exists $f \in R$ such that $f a_{i}=b_{i}, i=1, \cdots, j$.

We now obtain

TheOREM 4.5. Suppose $T(G)$ satisfies the ascending chain condition. Then $G$ is separable with linearly ordered type set if and only if $F(G)$ is fully 2-fold ct-transitive, and hence fully $k$-fold ct-transitive for every $k$.

Proof. The sufficiency is established in Proposition 4.3. Conversely, $F(G)$ is 2-fold $c t$-transitive and hence $k$-fold $c t$-transitive for every $k$, as was seen in Theorem 3.1. $F(G)$ is fully so according to $[1$, p. 80, Lemma 5.2] and [4, p. 611, Lemma 3.9].

We proceed to demonstrate that the hypotheses of Theorem 4.5 impose no countability restriction upon $T(G)$. This result is of interest in its own right.

5. Chains of types with the maximum condition. Somewhat surprisingly, an uncountable chain of types can satisfy the maximum 
condition, unlike chains of heights. For the sake of definiteness: by a height we mean a function from the set of all primes to the set of nonnegative integers union $\infty$; by a type we mean an equivalence class of heights under the standard equivalence relation on heights $[7, \S 85]$; by a representation of a type $\tau$ we mean a choice of a height $h \in \tau$.

LEMMA 5.1. Every countable chain of types can be represented by a chain of heights.

Proof. Let $\tau_{1}, \tau_{2}, \cdots$ be an enumeration of a chain of types and let $h_{1}$ be a height in $\tau_{1}$. Suppose we have represented $\tau_{1}, \cdots, \tau_{n}$ by a chain of heights $h_{1}, \cdots, h_{n}$. Consider the three possibilities for $\tau_{n+1}$ :

(1) $\tau_{n+1}<\tau_{i}, i=1, \cdots, n$. Choose $h_{n+1} \in \tau_{n+1}$ so that $h_{n+1}<h_{i}$, $i=1, \cdots, n$.

(2) There exist $\tau_{i}$ and $\tau_{j}$ among $\tau_{1}, \cdots, \tau_{n}$ such that $\tau_{i}<\tau_{n+1}<\tau_{j}$; we take $\tau_{i}$ to be the greatest such type and $\tau_{j}$ the least such. Choose $h_{n+1} \in \tau_{n+1}$ so that $h_{i}<h_{n+1}<h_{j}$.

(3) $\tau_{n+1}>\tau_{i}, i=1, \cdots, n$. Choose $h_{n+1} \in \tau_{n+1}$ satisfying $h_{n+1}>$ $h_{i}, i=1, \cdots, n$.

We thus obtain a chain of heights $h_{i} \in \tau_{i}, i=1,2, \cdots$.

REMARK 5.2. In Lemma 5.1, the countability assumption is essential. It is not difficult to prove that any chain of heights which satisfies the maximum (or minimum) condition must be countable. There do, of course, exist uncountable chains of heights which represent distinct types.

To say that the types $\tau_{1}, \tau_{2}$ differ at an infinite number of primes means that $h_{1} \in \tau_{1}$ and $h_{2} \in \tau_{2}$ differ at infinitely many primes.

THEOREM 5.3. Let $\tau<\tau^{\prime \prime}$ be two types which differ at an infinite number of primes. Then there exists an uncountable chain of types satisfying the maximum condition which contains $\tau$ as its least element and $\tau^{\prime \prime}$ as its greatest element.

Proof. Choose a type $\tau^{\prime}$ so that $\tau<\tau^{\prime} \leqq \tau^{\prime \prime}$ and so that $\tau^{\prime}$ is finite wherever it differs from $\tau$. Let $\mathscr{C}$ denote the collection of all sets $C$ of types such that (1) $C$ is a chain satisfying the maximum condition; (2) $\tau^{\prime} \in C$; (3) $\tau<\sigma \leqq \tau^{\prime}$ for all $\sigma \in C$; (4) if $\sigma_{1}, \sigma_{2}$ are distinct elements of $C$, then $\sigma_{1}$ and $\sigma_{2}$ differ from each other and from $\tau$ at an infinite number of primes. Note that $\left\{\tau^{\prime}\right\} \in \mathscr{C}$.

For $C_{1}, C_{2} \in \mathscr{C}$, define $C_{1}<C_{2}$ if there exists $\sigma \in C_{2}$ such that $C_{1}=$ 
$\left\{\sigma^{\prime} \in C_{2}: \sigma<\sigma^{\prime}\right\}$. By standard arguments, under this partial ordering of $\mathscr{C}$, chains have upper bounds in $\mathscr{C}$. Zorn's Lemma then implies that $\mathscr{C}$ contains a maximal element $M$; observe that $M$ contains no least element. Suppose $M$ is countable and enumerate its elements $\tau_{1}, \tau_{2}, \cdots$. From Lemma 5.1, $M$ can be represented by a chain of heights $h_{1}, h_{2}, \cdots$, with $h_{i} \in \tau_{i}, i=1,2, \cdots$. For a fixed $h \in \tau$, we may assume $h_{i}>h$ for all $i$. We now define a height $f$ which represents a type $\sigma$ such that $\tau<\sigma<\tau_{i}, i=1,2, \cdots$, and such that $\sigma$ differs from $\tau$ and each $\tau_{i}$ at an infinite number of primes.

Set $m_{1}=1$, let $p_{n_{1}}$ be a prime such that $h_{m_{1}}\left(p_{n_{1}}\right)>h\left(p_{n_{1}}\right)$, and define $f(p)=h_{m_{1}}(p)$ for all primes $p, 2 \leqq p \leqq p_{n_{1}}$. Suppose now that $f$ has been defined for all primes $p, 2 \leqq p \leqq p_{n_{k}}$. Choose $m_{k+1}$ greater that $m_{k}$ so that $h_{m_{k+1}}<h_{i}$ for $1 \leqq i<m_{k+1}$ and let $p_{n_{k+1}}$ be a prime greater than $p_{n_{k}}$ such that $h_{m_{k+1}}\left(p_{n_{k+1}}\right)>h\left(p_{n_{k+1}}\right)$. Define $f(p)=h_{m_{k+1}}(p)$ for primes $p$ satisfying $p_{n_{k}}<p \leqq p_{n_{k+1}}$.

It is straightforward to verify that $f$ represents a type $\sigma$ having the properties claimed, so that $\{\sigma\} \cup M$ contradicts the maximality of $M$. Thus $M$ is uncountable and the chain $\{\tau\} \cup M \cup\left\{\tau^{\prime \prime}\right\}$ satisfies the maximum condition and contains $\tau, \tau^{\prime \prime}$ as its least, respectively, greatest element.

REMARK 5.4. (1) If $\tau$ and $\tau^{\prime \prime}$ are distinct types which differ at only finitely many primes, then any chain of types between $\tau$ and $\tau^{\prime \prime}$ is necessarily finite.

(2) The preceding arguments may be modified in an obvious fashion to prove the existence of uncountable well ordered type sets.

(3) Under the continuum hypothesis, any uncountable chain of types has the same cardinality, $2^{\mathrm{N}_{0}}$, as the set of all types.

\section{REFERENCES}

1. Reinhold Baer, Abelian groups without elements of finite order, Duke Math. J., 3 (1937), 68-122.

2. R. A. Beaumont and R. S. Pierce, Torsion-free rings, Illinois J. Math., 5 (1961), 61-98.

3. - Torsion free groups of rank two, Memoirs Amer. Math. Soc., 38 (1961).

4. E. F. Cornelius, Jr., A generalization of separable groups, Pacific J. Math., 39 (1971), 603-613.

5. — A sufficient condition for separability, submitted for publication.

6. L. Fuchs, Abelian groups, reprint, Internat. Series of Monographs of Pure and Appl. Math., Pergamon Press, New York, 1960.

7. - Infinite Abelian Groups, Vol. 2, Academic Press, New York, 1973.

8. Nathan Jacobson, Structure of rings, rev. ed., Amer. Math. Soc. Colloq. Publ., vol.

37, Amer. Math. Soc., Providence, R. I., 1964.

9. J. D. Reid, On quasi-decompositions of torsion free abelian groups, Proc. Amer. Math. Soc., 13 (1962), 550-554.

10. On the ring of quasi-endomorphisms of a torsion-free group, Topics in 
abelian groups, Scott, Foresman, Chicago, 1964, 51-68.

Received June 7, 1978

Wayne State University

DETROIT, MI 48202

Current Address: DYKEMA, GOSSETT, SPENCER, GOODNOW \& TRIGG 35th Floor, 400 Renaissance Center Detroit, MI 48243 



\section{PACIFIC JOURNAL OF MATHEMATICS}

\section{EDITORS}

RichaRd ARENS (Managing Editor)

University of California

Los Angeles, CA 90024

Charles W. Curtis

University of Oregon

Eugene, OR 97403

C. C. Moore

University of California

Berkeley, CA 94720

\section{J. DUGUNDJI}

Department of Mathematics

University of Southern California

Los Angeles, CA 90007

R. FinN and J. Milgram

Stanford University

Stanford, CA 94305

\section{E. F. BECKENBACH \\ B. H. NeumanN

\author{
UNIVERSITY OF BRITISH COLUMBIA \\ CALIFORNIA INSTITUTE OF TECHNOLOGY \\ UNIVERSITY OF CALIFORNIA \\ MONTANA STATE UNIVERSITY \\ UNIVERSITY OF NEVADA, RENO \\ NEW MEXICO STATE UNIVERSITY \\ OREGON STATE UNIVERSITY \\ UNIVERSITY OF OREGON
}

F. WOLF

K. Yoshida

\section{SUPPORTING INSTITUTIONS}

\author{
UNIVERSITY OF SOUTHERN CALIFORNIA \\ STANFORD UNIVERSITY \\ UNIVERSITY OF HAWAII \\ UNIVERSITY OF TOKYO \\ UNIVERSITY OF UTAH \\ WASHINGTON STATE UNIVERSITY \\ UNIVERSITY OF WASHINGTON
}

The Supporting Institutions listed above contribute to the cost of publication of this Journal, but they are not owners or publishers and have no responsibility for its content or policies.

Mathematical papers intended for publication in the Pacific Journal of Mathematics should be in typed form or offset-reproduced, (not dittoed), double spaced with large margins. Please do not use built up fractions in the text of the manuscript. However, you may use them in the displayed equations. Underline Greek letters in red, German in green, and script in blue. The first paragraph or two must be capable of being used separately as a synopsis of the entire paper. Items of the bibliography should not be cited there unless absolutely necessary, in which case they must be identified by author and journal, rather than by item number. Manuscripts, in triplicate, may be sent to any one of the editors. Please classify according to the scheme of Math. Reviews, Index to Vol. 39. All other communications should be addressed to the managing editor, or Elaine Barth, University of California, Los Angeles, California, 90024.

50 reprints to each author are provided free for each article, only if page charges have been substantially paid. Additional copies may be obtained at cost in multiples of 50 .

The Pacific Journal of Mathematics is issued monthly as of January 1966. Regular subscription rate: $\$ 72.00$ a year (6 Vols., 12 issues). Special rate: $\$ 36.00$ a year to individual members of supporting institutions.

Subscriptions, orders for numbers issued in the last three calendar years, and changes of address should be sent to Pacific Journal of Mathematics, P.O. Box 969, Carmel Valley, CA 93924, U.S.A. Older back numbers obtainable from Kraus Periodicals Co., Route 100, Millwood, NY 10546.

PUBLISHED BY PACIFIC JOURNAL OF MATHEMATICS, A NON-PROFIT CORPORATION

Printed at Kokusai Bunken Insatsusha (International Academic Printing Co., Ltd.). 8-8, 3-chome, Takadanobaba, Shinjuku-ku, Tokyo 160, Japan. 


\section{Pacific Journal of Mathematics \\ Vol. 79, No. $2 \quad$ June, 1978}

David R. Adams, Quasi-additivity and sets of finite $L^{p}$-capacity ........ 283

George M. Bergman and Warren Dicks, Universal derivations and universal

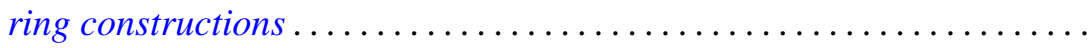

Robert F. Brown, Addendum to: "Fixed points of automorphisms of compact Lie groups".........................................

Eugene Frank Cornelius, Jr., Characterization of a class of torsion free

groups in terms of endomorphisms .......................

Andres del Junco, A simple measure-preserving transformation with trivial centralizer..................................... 357

Allan Lee Edmonds, Extending a branched covering over a handle ...... 363

Sjur Flam, A characterizaton of $\mathbf{R}^{2}$ by the concept of mild convexity .......

Claus Gerhardt, $L^{p}$-estimates for solutions to the instationary Navier-Stokes

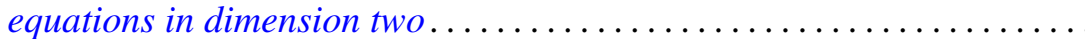

Kensaku Gomi, Finite groups with a standard subgroup isomorphic to

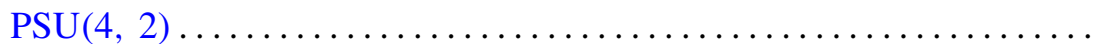

E. E. Guerin, A convolution related to Golomb's root function ........... 463

$\mathrm{H}$. B. Hamilton, Modularity of the congruence lattice of a commutative cancellative semigroup .................................

Stephen J. Haris, Complete reducibility of admissible representations over function fields.

Shigeru Itoh and Wataru Takahashi, The common fixed point theory of singlevalued mappings and multivalued mappings... ...

James E. Joseph, Multifunctions and graphs .............

Bruce Magurn, Images of $S K_{1} Z G$. .

Arnold Koster Pizer, A note on a conjecture of Hecke... .

Marlon C. Rayburn, Maps and h-normal spaces ...........

Barada K. Ray and Billy E. Rhoades, Corrections to: "Fixed-point theorems for mappings with a contractive iterate" ..............

Charles Irvin Vinsonhaler, Corrections to: "Torsion free abelian groups quasiprojective over their endomorphism rings. II". . . 